(1)

CrossMark

\title{
Functional ageing in fibrotic interstitial lung disease: the impact of frailty on adverse health outcomes
}

\author{
Sabina A. Guler (10 1,2,3, Joanne M. Kwan ${ }^{1,2}$, Janice M. Leung ${ }^{1,2}$, Nasreen Khalil ${ }^{4}$, \\ Pearce G. Wilcox ${ }^{1}$ and Christopher J. Ryerson ${ }^{1,2}$ \\ Affiliations: ${ }^{1}$ Dept of Medicine, University of British Columbia, Vancouver, BC, Canada. ${ }^{2}$ Centre for Heart Lung \\ Innovation, University of British Columbia, Vancouver, BC, Canada. ${ }^{3}$ Dept of Pulmonary Medicine, University \\ Hospital and University of Bern, Bern, Switzerland. ${ }^{4}$ The Lung Centre, Vancouver General Hospital, Vancouver, \\ BC, Canada. \\ Correspondence: Christopher J. Ryerson, St Paul's Hospital, 1081 Burrard St, Ward 8B, Vancouver, BC, \\ Canada, V6Z 1Y6. E-mail: chris.ryersonahli.ubc.ca
}

@ERSpublications

Frailty independently predicts adverse health outcomes in patients with fibrotic ILD; with functional ageing as the main driver of most age-related adverse health outcomes there is a need to recognise, prevent and treat frailty in this population $\mathrm{http}: / / \mathrm{bit} . \mathrm{ly} / 2 \mathrm{k} 81 \mathrm{rRc}$

Cite this article as: Guler SA, Kwan JM, Leung JM, et al. Functional ageing in fibrotic interstitial lung disease: the impact of frailty on adverse health outcomes. Eur Respir J 2020; 55: 1900647 [https://doi.org/ 10.1183/13993003.00647-2019].

\section{ABSTRACT}

Background: Accelerated biological and functional ageing is common in fibrotic interstitial lung disease (ILD); however, their impact on adverse health outcomes has not been evaluated in this population.

Methods: Patients were prospectively recruited from a specialised ILD clinic. Functional ageing was determined by frailty index and biological age by measurement of absolute telomere length (aTL) from patients' peripheral blood leukocytes. Adverse health outcomes included health-related quality of life (St George's Respiratory Questionnaire), number and length of respiratory and non-respiratory hospitalisations, medication tolerability and time to death or lung transplantation. Multivariable models were used to determine the risks and rates of adverse health outcomes associated with the frailty index and aTL.

Results: 540 patients with fibrotic ILD, including 100 with idiopathic pulmonary fibrosis (IPF), provided 749 frailty index assessments, with 189 patients providing blood samples. The frailty index was strongly associated with quality of life, rate of hospitalisation, time to hospital discharge and mortality, including adjustment for age, sex, disease severity and IPF diagnosis. Mortality prognostication was improved by the addition of the frailty index to commonly used clinical parameters and previously validated composite indices. Conversely, aTL was not associated with most adverse health outcomes. The effect of chronological age on outcomes was mediated primarily by the frailty index, and to a lesser extent by aTL. Conclusions: Functional ageing is associated with adverse health outcomes in patients with fibrotic ILD, indicating the need for consideration of the individual functional age into clinical decision-making.

This article has been revised according to the correction published in the March 2020 issue of the European Respiratory Journal.

This article has an editorial commentary: https://doi.org/13993003.02255-2019

This article has supplementary material available from erj.ersjournals.com

Received: 30 March 2019 | Accepted after revision: 01 Sept 2019

Copyright $\odot$ ERS 2020 


\section{Introduction}

Interstitial lung disease (ILD) is an increasingly common group of inflammatory and fibrotic disorders that damage the lung parenchyma. Fibrotic ILDs are typically associated with exercise limitation, reduced quality of life and early mortality. Many fibrotic ILDs, including idiopathic pulmonary fibrosis (IPF), increase in incidence with age and are thus frequently associated with comorbidities that increase the complexity of management and place substantial burden on the healthcare system [1-3].

The time-dependent gradual loss of physical and social functioning with ageing is typically accelerated in patients with chronic diseases [4], and many cellular and molecular hallmarks of ageing are frequently and prematurely observed in patients with fibrotic ILDs $[5,6]$. The accelerated functional ageing associated with fibrotic ILD is best represented by the concept of frailty, defined as the accumulation of age- and health-related deficits across physical, psychological and social systems [4, 7-9]. This diminished physiological reserve and increased vulnerability to minor stressors is common in fibrotic ILD [10-12], and may expose these patients to adverse health outcomes. Frailty has been associated with a higher risk of death in the general population [4, 9], in patients with chronic obstructive pulmonary disease [13] and in lung transplant candidates [14]. However, the risks associated with frailty as a proxy of accelerated functional ageing, and different impacts of functional, biological and chronological ageing in patients with fibrotic ILD have not been evaluated.

The goal of this study was therefore to determine the impact of functional ageing (i.e. frailty) on mortality, hospitalisations and quality of life in a large cohort of patients with fibrotic ILD. We used peripheral blood leukocyte telomere length as a proxy of biological ageing to further determine the specific role of functional ageing compared to biological and chronological ageing. Our prespecified central hypothesis was that frailty would independently predict adverse health outcomes in fibrotic ILD, thus indicating the central importance of functional ageing in the management of these patients.

\section{Methods}

\section{Study population and measurements}

Consecutive patients with a multidisciplinary diagnosis of fibrotic ILD were recruited from an outpatient ILD referral centre between July 2014 and July 2017, including patients with IPF [15], chronic hypersensitivity pneumonitis [16], unclassifiable ILD and connective tissue disease (CTD)-associated ILD [17]. All patients provided informed written consent (University of British Columbia ethics board approval H10-03099).

\section{Functional ageing: the frailty index}

Frailty, representing functional ageing, was measured using a self-reported frailty index consisting of 42 deficits, including 19 comorbidities and 23 deficits related to independence and self-care. Patients confirmed or denied the presence of each equally weighted deficit, as previously described [18]. The frailty index is calculated as the proportion of items present divided by the total number of surveyed items, expressed as a continuous variable between 0 and 1 . Surveyed deficits without a patient response were removed from the denominator ( $1.5 \%$ of all surveyed items). Frailty was defined as frailty index $>0.21$ [4]. Data from the Canadian Study of Health and Aging show a submaximal limit of the frailty index of $0.65 \pm 0.02$ in a general outpatient population, beyond which mortality is imminent [19]. We previously demonstrated that the frailty index has good internal consistency in fibrotic ILD and that frailty is more prevalent in patients with fibrotic ILD than in the general population [10]. The items within the frailty index were subcategorised into 19 items related to comorbidities (Co-FI), and 23 items related to independence and self-care (ISC-FI). The frailty index was completed at study entry, including both incident and prevalent diagnoses of ILD, and during follow-up when patients returned for routine clinical assessments. Frailty assessments that were completed within 6 months of a preceding frailty index calculation were excluded from analysis, in order to avoid overlapping observation periods.

\section{Biological ageing: leukocyte telomere length}

Blood leukocyte telomere length was measured in a random subgroup of consecutively recruited patients who consented to donate blood samples. We applied a modified version of the CAWTHON [20, 21] method for measurement of absolute telomere length (aTL) using quantitative real-time PCR. A detailed description is provided in the supplementary material.

\section{Outcome assessments}

Health-related quality of life was measured using the St George's Respiratory Questionnaire (SGRQ) without any modification. This 50-item patient-reported questionnaire was specifically developed for respiratory diseases [22], is frequently used in patients with ILD, and has recently been validated in CTD-associated ILD [23, 24]. The SGRQ includes three domains relating to symptoms, activity and impact, as well as a total score that ranges from 0 to 100 , with a higher score indicating worse quality of 
life. Non-elective respiratory-related hospitalisations, non-respiratory-related hospitalisations and cumulative number of days admitted to hospital within 6 months of each frailty assessment were identified from the medical record. Patients completing more than one frailty assessment contributed multiple non-overlapping 6-month follow-up intervals during the study period. Time to death, lung transplantation or censoring was calculated from the date of the first frailty assessment. The medical record was used to identify adverse effects related to prevalent ILD pharmacotherapy that occurred within 6 months of each frailty assessment. Medication adverse reactions (MAR) were defined according to the World Health Organization as an unintended or noxious response to a drug that occurs at doses normally used in humans [25]. MARs were specified as effects that resulted in a dose reduction, purposeful treatment interruption for $>1$ day, or treatment discontinuation for $>14$ days [25].

\section{Other measurements}

Demographics and baseline characteristics were collected from the clinical record, including age, sex, body mass index and smoking history. Pulmonary function tests were performed using established protocols [26, 27]. All clinical measurements were completed within 3 months of the frailty assessment.

\section{Statistical analysis}

Data structure was hierarchical with multiple frailty index and covariate measurements per patient at time intervals of $\geqslant 6$ months. The primary mortality end-point was time to the composite of death or lung transplantation. Other outcomes were assessed within the 6-month time periods after every frailty index assessment. Time to death or lung transplantation and time to hospital discharge were each analysed using Cox proportional hazards regression models. Discrimination was measured using Harrell's C-statistic. In a prespecified sensitivity analysis we used competing risk regression according to FINE and GRAY [28], with death and lung transplantation as competing risks. Rate of hospitalisations, probabilities for MAR and associations with SGRQ were modelled using generalised mixed effects models accounting for intrapatient correlation with random intercepts. Fixed effects included frailty index, telomere length and potential confounders with either conceptual importance (age, sex) or a statistically relevant relationship to the outcome. These models were fitted for linear, binomial and Poisson distribution families according to the outcomes of interest. Mediation of the effect of chronological age on adverse health outcomes by either functional age (frailty index) or by biological age (aTL) was explored using causal mediation analysis. Estimates for the average causal mediation effect/average total effect for an increase in chronological age by 1 year were reported. A directed acyclic graph is provided in supplementary figure S1. Model specification, assumption testing and specific statistical programmes used are reported in the supplementary material. A two-sided $\mathrm{p}<0.05$ was used to indicate statistical significance for all comparisons. Data were analysed using $\mathrm{R}$ version 3.5.1 [29].

\section{Results}

\section{Patient characteristics}

The 540 patients provided a total of 749 frailty assessments with a median (interquartile range (IQR)) interval of 10.8 (6.2-17.7) months between assessments in patients who completed more than one frailty questionnaire (table 1). Diagnoses included IPF $(n=100)$, systemic sclerosis-associated ILD ( $\mathrm{n}=109)$, other CTD-ILD ( $\mathrm{n}=118)$, chronic hypersensitivity pneumonitis $(\mathrm{n}=47)$ and 39 "other" ILDs. It was not possible to assign a confident diagnosis to 127 patients, who were designated as "unclassifiable" ILD. At baseline, $42 \%$ of males and $56 \%$ of females were classified as frail. The median (IQR) frailty index for the entire cohort was $0.214(0.095-0.333)$, the Co-FI was $0.158(0.052-0.211)$ and the ISC-FI was $0.261(0.087-0.435)$. Females had accelerated functional ageing with higher frailty scores compared to males, as did patients with non-IPF ILDs compared to patients with IPF (table 2).

\section{Health-related quality of life}

Frailty severity was correlated with worse quality of life on unadjusted analysis and with adjustment for age, sex, ILD severity and IPF diagnosis (table 3). This was present for pairwise associations of all frailty measures (frailty index, Co-FI, ISC-FI) with all SGRQ domains.

\section{Hospitalisations}

There were 231 non-elective hospitalisations within the 749 observation periods from the 540 patients, including 131 respiratory-related hospitalisations. Over the entire study period, 459 patients had no hospitalisations and 81 patients had between one and five hospitalisations. The median cumulative number of days admitted to hospital within 6 months of frailty assessment was 5 (2-14) days among those periods with at least one hospitalisation. Patients with more severe frailty had a higher rate of all-cause and respiratory-related hospitalisations and longer time to hospital discharge on unadjusted and adjusted analysis, with these associations primarily driven by the ISC-FI component of frailty (table 3 and figure 1). 


\begin{tabular}{lcc}
$\begin{array}{l}\text { TABLE } 1 \text { Baseline characteristics of males and females with fibrotic interstitial lung diseases } \\
\text { (ILDs) }\end{array}$ & Males & Females \\
\hline & 232 & 308 \\
\hline Subjects & & \\
Demographics & $67.4 \pm 10.2$ & $62.8 \pm 12.4$ \\
Age years & $28.5 \pm 5.0$ & $27.3 \pm 6.0$ \\
Body mass index $\mathrm{kg} \cdot \mathrm{m}^{-2}$ & $128(55)$ & $144(47)$ \\
Ever-smoker & $24.2(9.5-37)$ & $16.0(1.8-25)$ \\
Smoked pack-years ${ }^{\#}$ & & \\
ILD severity & $73.0 \pm 19.0$ & $75.1 \pm 22.1$ \\
FVC \% pred & $74.9 \pm 18.3$ & $77.1 \pm 23.3$ \\
FEV $\%$ pred & $52.9 \pm 18.8$ & $52.9 \pm 18.7$ \\
DLCo \% pred & & \\
Quality of life & $42.3 \pm 22.8$ & $43.3 \pm 22.2$ \\
SGRQ total & $53.3 \pm 26.6$ & $58.1 \pm 23.6$ \\
SGRQ activity & $51.2 \pm 23.2$ & $48.0 \pm 24.2$ \\
SGRQ symptom & $32.8 \pm 22.8$ & $33.8 \pm 23.5$ \\
SGRQ impact & &
\end{tabular}

Data are presented as $\mathrm{n}$, mean $\pm \mathrm{SD}, \mathrm{n}(\%)$ or median (interquartile range). FVC: forced vital capacity; $\%$ pred: \% predicted; $\mathrm{FEV}_{1}$ : forced expiratory volume in $1 \mathrm{~s} ; D_{\mathrm{LCO}}$ : diffusing capacity of the lung for carbon monoxide; SGRQ: St George's Respiratory Questionnaire. "\#: in ever-smokers.

On unadjusted analysis, frail patients had more than double the rate of all-cause and respiratory-related hospitalisations with significantly greater risk for prolonged hospital stay. All but the association with respiratory-related hospitalisations remained statistically significant on adjusted analysis.

\section{Survival}

Over the median (IQR) follow-up time of 17 (9.2-26.7) months, 81 patients died and 14 patients underwent lung transplantation. Patients with advanced functional ageing, hence classified as frail, had significantly worse 1-, 2- and 3-year transplant-free survival of $86 \%, 77 \%$ and $73 \%$, respectively, compared to non-frail patients $(94 \%, 91 \%$ and $87 \%$, respectively; $\mathrm{p}<0.001)$ (figure 2 ). Higher frailty index and ISC-FI were associated with time to death or transplant on unadjusted analysis and with adjustment for age, sex, IPF diagnosis and ILD severity (table 3), and with adjustment for the composite gender-agephysiology-ILD index (data not shown) [30]. The prognostic ability of the models increased substantially when frailty index was added to age and sex, with a marginal and probably clinically non-relevant increase of the C-index when frailty index was added to a multivariate model including age, sex, ILD severity and IPF diagnosis (table 4). Consistent results were obtained using a prespecified sensitivity analysis that considered death and lung transplantation as competing risks. The submaximal limit of the frailty index was 0.67 , corresponding to the 99th percentile. Stratification by IPF and CTD-ILD diagnoses revealed largely unchanged findings (data not shown).

TABLE 2 Frailty and telomere length in males and females with idiopathic pulmonary fibrosis (IPF) and non-IPF fibrotic interstitial lung diseases (ILDs)

\begin{tabular}{lcccc} 
& Males & Females & IPF & Non-IPF ILD \\
\hline Subjects & 232 & 308 & 100 & 440 \\
Frailty index & $0.167(0.071-0.286)$ & $0.238(0.120-0.358)$ & $0.167(0.092-0.288)$ & $0.214(0.095-0.333)$ \\
Co-FI & $0.105(0.053-0.211)$ & $0.158(0.105-0.263)$ & $0.111(0.097-0.211)$ & $0.158(0.055-0.214)$ \\
ISC-FI & $0.217(0.043-0.384)$ & $0.304(0.087-0.478)$ & $0.174(0.077-0.391)$ & $0.261(0.087-0.435)$ \\
Frail (frailty index $>\mathbf{0 . 2 1 )}$ & $98(42)$ & $174(56)$ & $39(39)$ & $233(53)$ \\
Prefrail (frailty index $\mathbf{0 . 1 - 0 . 2 1 )}$ & $58(25)$ & $61(20)$ & $28(28)$ & $91(21)$ \\
aTL kbp per genome & $171.1 \pm 45.4$ & $185.6 \pm 45.8$ & $163.3 \pm 40.7$ & $183.2 \pm 46.7$ \\
\hline
\end{tabular}

Data are presented as $\mathrm{n}$, median (interquartile range), $\mathrm{n}(\%)$ or mean $\pm \mathrm{SD}$. Co-FI: comorbidity frailty index; ISC-FI: independence and self-care frailty index; aTL: absolute telomere length. ${ }^{\#}$ : aTL was calculated for a subgroup of patients with available blood samples (sample size: males $n=87$, females $n=102$, IPF $n=41$, non-IPF ILD $n=148$ ). 
TABLE 3 Association of frailty with outcomes in fibrotic interstitial lung disease (ILD)

\begin{tabular}{c} 
Unadjusted analysis \\
\hline $\begin{array}{c}\text { Coefficient/IRR/HR p-value } \\
(95 \% \mathrm{CI})\end{array}$
\end{tabular}

Adjusted for age, sex, FVC $\%$ pred, $D_{\text {Lco }} \%$ pred, IPF

(95\% Cl)

Coefficient/IRR/HR p-value (95\% CI)

\begin{tabular}{|c|c|c|c|c|}
\hline \multicolumn{5}{|c|}{$\begin{array}{l}\text { Health-related quality of life } \\
\text { (SGRQ) }\end{array}$} \\
\hline Frailty index & $7.01(6.04-7.98)$ & $<0.0001$ & $5.83(4.81-6.84)$ & $<0.0001$ \\
\hline $\mathrm{Co}-\mathrm{Fl}$ & $3.49(2.25-4.72)$ & $<0.0001$ & $4.00(2.79-5.22)$ & $<0.0001$ \\
\hline ISC-FI & $5.25(4.58-5.92)$ & $<0.0001$ & $4.41(3.68-5.15)$ & $<0.0001$ \\
\hline Frail & $21.6(18.0-25.4)$ & $<0.0001$ & $18.0(14.1-21.7)$ & $<0.0001$ \\
\hline \multicolumn{5}{|c|}{ Rate of all-cause hospitalisations } \\
\hline Frailty index & $1.03(1.02-1.04)$ & $<0.0001$ & $1.03(1.01-1.04)$ & $<0.0001$ \\
\hline $\mathrm{Co}-\mathrm{Fl}$ & $1.02(1.00-1.03)$ & 0.01 & $1.03(1.01-1.04)$ & 0.003 \\
\hline ISC-FI & $1.02(1.01-1.02)$ & $<0.0001$ & $1.02(1.01-1.03)$ & 0.0001 \\
\hline Frail & $2.25(1.38-3.47)$ & $<0.0001$ & $1.97(1.32-3.06)$ & 0.002 \\
\hline \multicolumn{5}{|c|}{$\begin{array}{l}\text { Rate of respiratory-related } \\
\text { hospitalisations }\end{array}$} \\
\hline Frailty index & $1.03(1.01-1.04)$ & 0.0003 & $1.02(1.01-1.04)$ & 0.02 \\
\hline $\mathrm{Co}-\mathrm{Fl}$ & $1.01(0.99-1.03)$ & 0.24 & $1.02(1.00-1.04)$ & 0.06 \\
\hline ISC-FI & $1.02(1.01-1.03)$ & $<0.0001$ & $1.01(1.00-1.03)$ & 0.03 \\
\hline Frail & $2.01(1.25-3.34)$ & 0.003 & $1.63(0.79-2.43)$ & 0.09 \\
\hline \multicolumn{5}{|c|}{ Time to hospital discharge } \\
\hline Frailty index & $1.02(1.00-1.03)$ & 0.005 & $1.02(1.00-1.03)$ & 0.009 \\
\hline $\mathrm{Co}-\mathrm{Fl}$ & $1.01(0.99-1.02)$ & 0.48 & $1.01(0.99-1.03)$ & 0.19 \\
\hline ISC-FI & $1.01(1.01-1.02)$ & 0.001 & $1.01(1.00-1.02)$ & 0.004 \\
\hline Frail & $1.56(1.55-1.57)$ & 0.01 & $1.35(1.32-1.38)$ & 0.048 \\
\hline \multicolumn{5}{|l|}{ Time to death } \\
\hline Frailty index & $1.03(1.02-1.04)$ & $<0.0001$ & $1.02(1.00-1.04)$ & 0.02 \\
\hline $\mathrm{Co}-\mathrm{Fl}$ & $1.01(0.99-1.02)$ & 0.46 & $1.02(0.99-1.04)$ & 0.26 \\
\hline ISC-FI & $1.03(1.02-1.04)$ & $<0.0001$ & $1.02(1.00-1.03)$ & 0.02 \\
\hline Frail & $2.64(2.31-3.02)$ & $<0.0001$ & $1.77(1.50-2.08)$ & 0.03 \\
\hline
\end{tabular}

Estimates are per 0.01-unit change in frailty index, except for health-related quality of life where the coefficient represents the change in St George's Respiratory Questionnaire (SGRQ) per square root change in frailty index. Frail is defined as frailty index $>0.21$. FVC: forced vital capacity; $\%$ pred: $\%$ predicted; $D_{\text {Lco: }}$ : diffusing capacity of the lung for carbon monoxide; IPF: diagnosis of idiopathic pulmonary fibrosis; IRR: incidence rate ratio; HR: hazard ratio; $\mathrm{Co}-\mathrm{FI}$ : comorbidity frailty index; ISC-FI: independence and self-care frailty index.
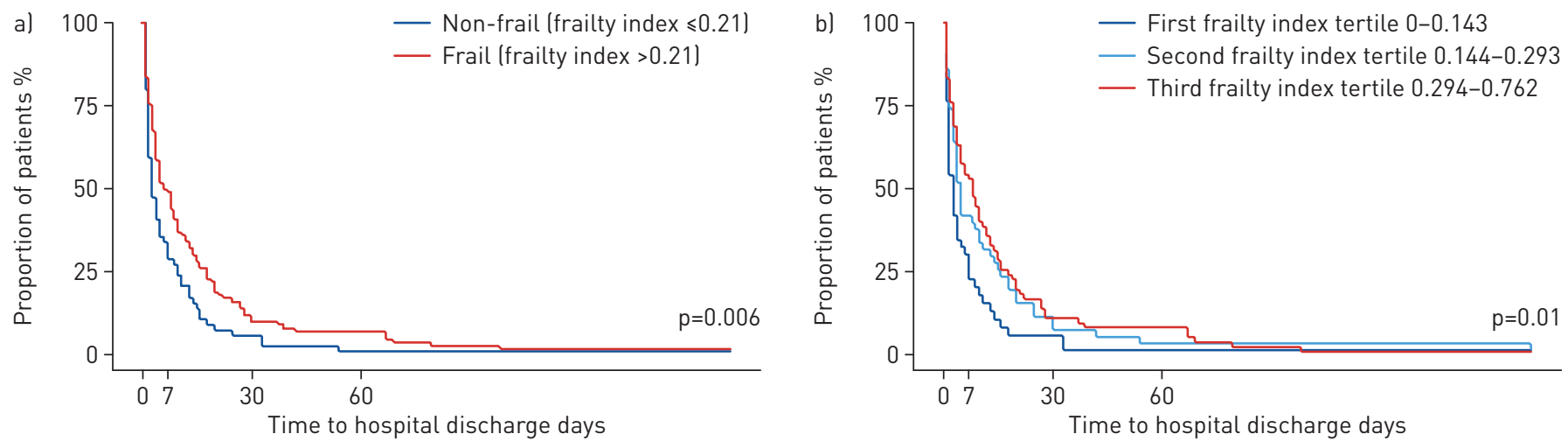

FIGURE 1 Time to hospital discharge a) in non-frail and frail patients and b) by tertiles of the frailty index. Survival curves from Cox proportional hazard models adjusting for age and sex. 

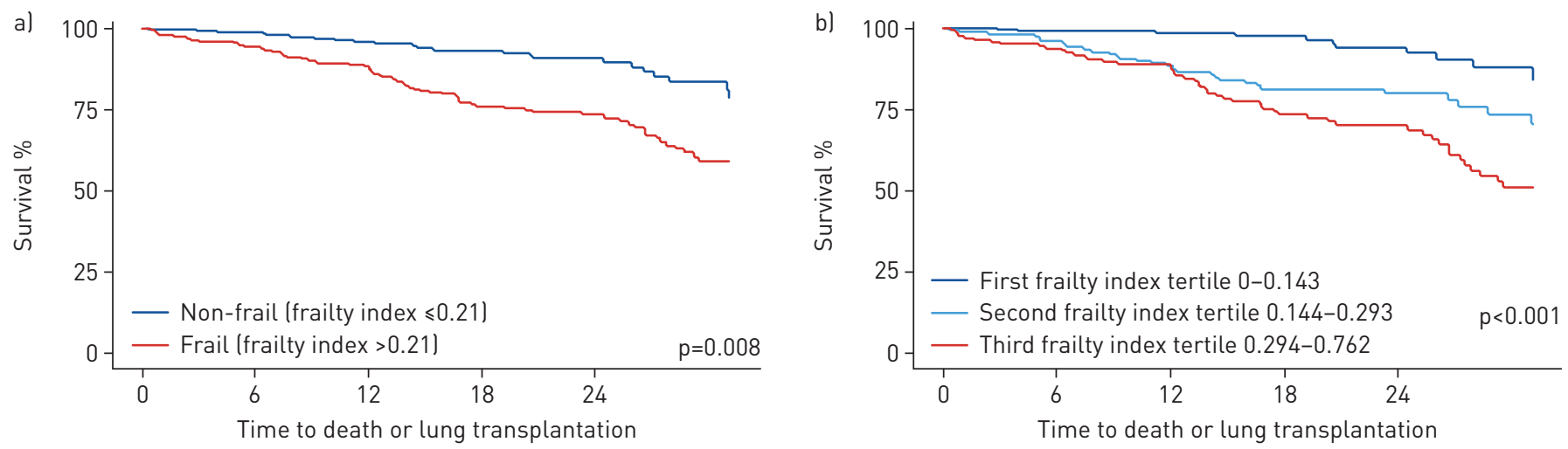

FIGURE 2 Survival a) in non-frail and frail patients and b) by tertiles of the frailty index. Survival curves from Cox proportional hazard models adjusting for age and sex.

\section{Medication adverse reactions}

The 239 (44\%) patients treated with ILD-specific medications had a higher frailty index $(0.244$ versus $0.191, \mathrm{p}=0.005)$ compared to untreated patients. In patients already on ILD-specific medication at the time of assessment, future dose reductions and treatment discontinuation both occurred in $3 \%$ of immunosuppressive treatments within 6 months of the frailty index assessment, whereas $12 \%$ of antifibrotic therapies resulted in MAR leading to treatment discontinuation. Within the subsequent 6-month observation period after frailty assessment, patients classified as frail were more likely to have a MAR resulting in dose reductions on unadjusted analysis (OR 11.3, 95\% CI 1.01-127; p=0.049); however, this analysis was underpowered and this association lost statistical significance with adjustment for age, sex, ILD severity and IPF diagnosis (OR 7.03, 95\% CI 0.62-79.8; $\mathrm{p}=0.12$ ).

\section{Impact of telomere length on outcomes}

Baseline characteristics of the telomere subgroup were similar to the full cohort (supplementary table S2). aTL as a proxy of biological ageing showed no correlation with functional ageing (frailty index) in the subcohort of the 189 patients who had provided a blood sample at the time of frailty assessment $(r=0.04$, $\mathrm{p}=0.57)$, although there was moderate correlation of chronological and biological age $(\mathrm{r}=-0.36, \mathrm{p}<0.001)$. aTL did not predict survival or quality of life on unadjusted analysis; however, there was a $6 \%$ (incidence rate ratio (IRR), 95\% CI 0.99-1.12; $\mathrm{p}=0.056)$ and $9 \%($ IRR, 95\% CI 1.01-1.17; $\mathrm{p}=0.03$ ) higher rate of all-cause and respiratory-related hospitalisations for each $10 \mathrm{kpb}$ per genome decrease in aTL. These associations were not maintained with adjustment for age and sex (table 5).

\section{Causal mediation analysis}

An exploratory causal mediation analysis showed that the overall effect of chronological age on 2-year survival (per 10-year increase OR 2.41, 95\% CI 1.37-7.09; $\mathrm{p}=0.01$ ) was mediated largely by functional age (frailty index indirect/total effect 53\%), whereas a model including aTL as mediator showed that a smaller fraction of the effect of chronological age on outcomes was mediated by biological age (aTL indirect/total effect $31 \%$ ). Similarly, the increased rate of all-cause hospitalisations in older patients (per 10-year increase

\begin{tabular}{|c|c|c|c|c|}
\hline & Without FI士sE & $\mathrm{FI} \pm \mathrm{SE}$ & Co-FlıSE & ISC-FI士sE \\
\hline Unadjusted & & $0.679 \pm 0.028$ & $0.528 \pm 0.032$ & $0.709 \pm 0.026$ \\
\hline \multicolumn{5}{|l|}{ Adjusted } \\
\hline Age, sex & $0.648 \pm 0.028$ & $0.718 \pm 0.028$ & $0.650 \pm 0.028$ & $0.756 \pm 0.026$ \\
\hline Age, sex, FVC, $D_{\text {Lco }}$ & $0.853 \pm 0.021$ & $0.860 \pm 0.021$ & $0.853 \pm 0.022$ & $0.866 \pm 0.020$ \\
\hline Age, sex, FVC, $D_{\text {LCo, IPF diagnosis }}$ & $0.857 \pm 0.021$ & $0.861 \pm 0.020$ & $0.857 \pm 0.021$ & $0.866 \pm 0.020$ \\
\hline
\end{tabular}

Data shown are optimism-corrected estimates of the Harrell's C-index, obtained by bootstrap resampling with 200 repetitions. FI: frailty index; Co-FI: comorbidity frailty index; ISC-FI: independence and self-care frailty index; FVC: forced vital capacity; $D_{\mathrm{LCO}}$ : diffusing capacity of the lung for carbon monoxide; IPF: idiopathic pulmonary fibrosis. 


\begin{tabular}{|c|c|c|c|c|}
\hline & \multicolumn{2}{|c|}{ Unadjusted analysis } & \multicolumn{2}{|c|}{ Adjusted for age and sex } \\
\hline & $\begin{array}{l}\text { HR/IRR/coefficient } \\
\text { (95\% CI) }\end{array}$ & p-value & $\begin{array}{l}\text { HR/IRR/coefficient } \\
\text { (95\% CI) }\end{array}$ & p-value \\
\hline Mortality & $0.92(0.80-1.04)$ & 0.21 & $0.96(0.83-1.12)$ & 0.60 \\
\hline All-cause hospitalisations & $0.94(0.88-1.01)$ & 0.06 & $0.96(0.89-1.03)$ & 0.25 \\
\hline $\begin{array}{l}\text { Respiratory-related } \\
\text { hospitalisations }\end{array}$ & $0.91(0.83-0.99)$ & 0.03 & $0.99(0.98-1.01)$ & 0.19 \\
\hline Time to hospital discharge & $1.01(0.94-1.05)$ & 0.83 & $0.99(0.92-1.06)$ & 0.70 \\
\hline $\begin{array}{l}\text { Health-related quality of life } \\
\text { (SGRQ) }\end{array}$ & $0.27(-0.50-1.04)$ & 0.49 & $0.31(-0.53-1.16)$ & 0.47 \\
\hline
\end{tabular}

Data shown are per $10 \mathrm{kbp}$ per genome change in telomere length. HR: hazard ratio; IRR: incidence rate ratio; SGRQ: St George's Respiratory Questionnaire.

IRR $1.35,95 \%$ CI 1.04-1.77; $\mathrm{p}=0.03$ ) was mainly mediated by functional age (frailty index indirect/total effect $43 \%$ ), and to a lesser extent by biological age (aTL indirect/total effect $22 \%$ ). Conversely, the effect of chronological age on respiratory-related hospitalisations (per 10-year increase IRR 1.54, 95\% CI 1.08 2.26; $\mathrm{p}=0.02$ ) was primarily mediated by biological age (aTL indirect/total effect $45 \%$ versus frailty index indirect/total effect 13\%). Robustness of the models to unmeasured confounding between the mediator and the outcome was confirmed as previously suggested and described in the supplementary methods [31].

\section{Discussion}

We evaluated chronological, functional and biological ageing in this large prospective cohort of patients with fibrotic ILD to show that frailty predicts adverse health outcomes and is a clinically relevant concept representing functional age in this population. Beyond its significant association with mortality, we show that frailty is associated with worse quality of life, a twofold higher rate of hospitalisations and longer hospital stay. Although underpowered, our findings also suggest that frailty may predict medication side effects and intolerance. Overall, it appears that in this cohort, functional ageing is prognostically more important than biological ageing.

Multidimensional mortality risk prediction models that consider demographic and lung function have a better discriminative ability compared to single predictor variables in patients with ILD [30, 32-34]; however, these are still suboptimal. There is increasing evidence on the impact of comorbidities and accelerated biological ageing on survival of patients with $\operatorname{ILD}[35,36]$, and measures of overall health state such as frailty may provide additional prognostic information. Using several clinically relevant outcomes, this is the first study showing that functional ageing provides prognostic information beyond that of commonly used clinical parameters in patients with ILD. A frailty index of 0.65 is the submaximal limit to the proportion of deficits that can be accumulated by elderly community-dwelling individuals before death is probably imminent [19]. Similarly, we show that the $99 \%$ limit to the frailty index was 0.67 in our younger cohort, consistent with previous observations that the frailty index limit is independent of chronological age. Combined with the reduced medication tolerance in frail patients, this finding suggests that patients approaching this submaximal limit should have their goals of care carefully reassessed, potentially changing to a palliative strategy that prioritises symptom management rather than continuation of potentially toxic ILD pharmacotherapy.

The role of ageing as a complex biosocial process is increasingly recognised in ILD [6]. To our knowledge, this is the first study investigating the clinical importance of functional, chronological and biological aspects of ageing in a single cohort of patients with fibrotic ILD, showing that functional ageing represented by the frailty index is overall more important than chronological and biological ageing for prognostication in this population. We explored potential causal pathways mediated by functional and biological ageing to show that functional ageing (i.e. frailty) drives $>50 \%$ of the overall age effect on mortality, whereas biological ageing (i.e. aTL) accounts for $\sim 30 \%$. Similarly, functional ageing was the primary driver of all-cause hospitalisations, while biological ageing was the main driver of respiratory-related hospitalisations. These findings suggest that respiratory-related hospitalisations may be predominantly caused by biological reasons such as ILD worsening (e.g. exacerbation, progressive hypoxaemia, chronic inflammation, catabolic metabolism), whereas the reasons for all-cause hospitalisations are usually more complex (e.g. decompensation of the social support system). 
Causal mediation analysis requires some assumptions that we addressed in sensitivity analyses, but there are no cut-off values to judge the robustness these analyses, and inferences from these models need to be confirmed in future studies. We had a relatively small size of some patient subgroups, which limited the power of subgroup analyses, particularly for telomere length analyses; however, our diverse cohort allows generalisation to a larger population of patients with fibrotic ILD. Despite the growing awareness of accelerated functional ageing as a public health problem, there is no agreement on a single definition of frailty [37]. Based on the importance of physical functionality for independence in daily living, the FRIED et al. [8] frailty phenotype, for example, includes a measure of physical performance. We used the frailty index, which is a simple measure that does not include any physical testing $[4,18,38]$, suggesting that physicians can easily apply many of these concepts in patient care. Self-reported tools such as the frailty index inherently incorporate a patient's self-efficacy and perspective on his/her own deficits. This reliance on individual reporting potentially introduced heterogeneity to our findings, but our large study cohort nevertheless allowed demonstration of statistically significant associations of functional ageing with adverse health outcomes.

\section{Conclusion}

This novel identification of functional ageing as the main driver of quality of life and most age-related adverse outcomes in fibrotic ILD emphasises the importance of recognising, preventing and treating frailty in this population. These findings suggest the need for integration of the individual functional age in clinical decision-making and prognostication in these patients. Future studies are needed to evaluate the utility of frailty in clinical decision algorithms and to investigate the effectiveness of specific treatment approaches to frailty in patients with fibrotic ILD.

Acknowledgements: The authors would like to acknowledge the patients of the St Paul's Hospital ILD Clinic, who allowed us to conduct this research in an effort to improve the lives of patients with ILD.

This work has been presented in the form of two abstracts at the American Thoracic Society International Conference 2018 in San Diego, and at the European Respiratory Society Congress 2018 in Paris.

Author contributions: C.J. Ryerson takes responsibility for the content of the manuscript, including the data and analysis and is guarantor of this paper. S.A. Guler and C.J. Ryerson contributed to the conception and design of the study, and acquisition, analysis and interpretation of the data. J.M. Kwan, J.M. Leung, N. Khalil and P.G. Wilcox contributed to the acquisition and interpretation of the data. All authors revised the manuscript for important intellectual content and provided final approval of the version to be published.

Support statement: This study was co-funded by the British Columbia Lung Association and InterMune/Hoffmann-La Roche Inc. Neither sponsor had input into the study design, analysis, interpretation of results, or the presentation of findings. Funding information for this article has been deposited with the Crossref Funder Registry.

Conflict of interest: None declared.

\section{References}

1 Navaratnam V, Fogarty AW, Glendening R, et al. The increasing secondary care burden of idiopathic pulmonary fibrosis: hospital admission trends in England from 1998 to 2010. Chest 2013; 143: 1078-1084.

2 Selman M, Pardo A. Revealing the pathogenic and aging-related mechanisms of the enigmatic idiopathic pulmonary fibrosis. An integral model. Am J Respir Crit Care Med 2014; 189: 1161-1172.

3 Navaratnam V, Fleming KM, West J, et al. The rising incidence of idiopathic pulmonary fibrosis in the U.K. Thorax 2011; 66: 462-467.

4 Rockwood K, Song X, Mitnitski A. Changes in relative fitness and frailty across the adult lifespan: evidence from the Canadian National Population Health Survey. CMAJ 2011; 183: E487-E494.

5 López-Otín C, Blasco MA, Partridge L, et al. The hallmarks of aging. Cell 2013; 153: 1194-1217.

6 Pardo A, Selman M. Lung fibroblasts, aging, and idiopathic pulmonary fibrosis. Ann Am Thorac Soc 2016; 13: Suppl. 5, S417-S421.

7 Searle SD, Mitnitski A, Gahbauer EA, et al. A standard procedure for creating a frailty index. BMC Geriatr 2008, 8: 24 .

8 Fried LP, Tangen CM, Walston J, et al. Frailty in older adults: evidence for a phenotype. J Gerontol A Biol Sci Med Sci 2001; 56: M146-M156.

9 Song X, Mitnitski A, Rockwood K. Prevalence and 10-year outcomes of frailty in older adults in relation to deficit accumulation. J Am Geriatr Soc 2010; 58: 681-687.

10 Milne KM, Kwan JM, Guler S, et al. Frailty is common and strongly associated with dyspnoea severity in fibrotic interstitial lung disease. Respirology 2017; 22: 728-734.

11 Guler SA, Kwan JM, Winstone TA, et al. Severity and features of frailty in systemic sclerosis-associated interstitial lung disease. Respir Med 2017; 129: 1-7.

12 Sheth JS, Xia M, Murray S, et al. Frailty and geriatric conditions in older patients with idiopathic pulmonary fibrosis. Respir Med 2019; 148: 6-12.

13 Galizia G, Cacciatore F, Testa G, et al. Role of clinical frailty on long-term mortality of elderly subjects with and without chronic obstructive pulmonary disease. Aging Clin Exp Res 2011; 23: 118-125.

14 Singer JP, Diamond JM, Gries CJ, et al. Frailty phenotypes, disability, and outcomes in adult candidates for lung transplantation. Am J Respir Crit Care Med 2015; 192: 1325-1334. 
15 Raghu G, Collard HR, Egan JJ, et al. An official ATS/ERS/JRS/ALAT statement: idiopathic pulmonary fibrosis: evidence-based guidelines for diagnosis and management. Am J Respir Crit Care Med 2011; 183: 788-824.

16 Travis WD, Costabel U, Hansell DM, et al. An official American Thoracic Society/European Respiratory Society statement: update of the international multidisciplinary classification of the idiopathic interstitial pneumonias. Am J Respir Crit Care Med 2013; 188: 733-748.

17 van den Hoogen F, Khanna D, Fransen J, et al. 2013 classification criteria for systemic sclerosis: an American College of Rheumatology/European League Against Rheumatism collaborative initiative. Ann Rheum Dis 2013; 72: $1747-1755$.

18 Mitnitski AB, Mogilner AJ, Rockwood K. Accumulation of deficits as a proxy measure of aging. Scientific World Journal 2001; 1: 323-336.

19 Rockwood K, Mitnitski A. Limits to deficit accumulation in elderly people. Mech Ageing Dev 2006; 127: 494-496.

20 Cawthon RM. Telomere measurement by quantitative PCR. Nucleic Acids Res 2002; 30: e47.

21 O'Callaghan NJ, Fenech M. A quantitative PCR method for measuring absolute telomere length. Biol Proced Online 2011; 13: 3 .

22 Jones PW, Quirk FH, Baveystock CM. The St George's Respiratory Questionnaire. Respir Med 1991; 85: Suppl. B, 25-31.

23 Yorke J, Jones PW, Swigris JJ. Development and validity testing of an IPF-specific version of the St George's Respiratory Questionnaire. Thorax 2010; 65: 921-926.

24 Suzuki A, Kondoh Y, Swigris JJ, et al. Performance of the St George's Respiratory Questionnaire in patients with connective tissue disease-associated interstitial lung disease. Respirology 2018; 23: 851-859.

25 Edwards IR, Aronson JK. Adverse drug reactions: definitions, diagnosis, and management. Lancet 2000; 356 $1255-1259$

26 Macintyre N, Crapo RO, Viegi G, et al. Standardisation of the single-breath determination of carbon monoxide uptake in the lung. Eur Respir J 2005; 26: 720-735.

27 Wanger J, Clausen JL, Coates A, et al. Standardisation of the measurement of lung volumes. Eur Respir J 2005; 26 : 511-522.

28 Fine JP, Gray RJ. A proportional hazards model for the subdistribution of a competing risk. J Am Stat Assoc 1999; 94: 496-509.

29 R Core Team. R: A Language and Environment for Statistical Computing. Vienna, Austria, R Foundation for Statistical Computing, 2016.

30 Ryerson CJ, Vittinghoff E, Ley B, et al. Predicting survival across chronic interstitial lung disease: the ILD-GAP model. Chest 2014; 145: 723-728.

31 Imai K, Keele L, Yamamoto T. Identification, inference and sensitivity analysis for causal mediation effects. Statist Sci 2010; 25: 51-71.

32 Ley B, Ryerson CJ, Vittinghoff E, et al. A multidimensional index and staging system for idiopathic pulmonary fibrosis. Ann Intern Med 2012; 156: 684-691.

33 Wells AU, Desai SR, Rubens MB, et al. Idiopathic pulmonary fibrosis: a composite physiologic index derived from disease extent observed by computed tomography. Am J Respir Crit Care Med 2003; 167: 962-969.

34 Morisset J, Vittinghoff E, Lee BY, et al. The performance of the GAP model in patients with rheumatoid arthritis associated interstitial lung disease. Respir Med 2017; 127: 51-56.

35 Hyldgaard C, Hilberg O, Bendstrup E. How does comorbidity influence survival in idiopathic pulmonary fibrosis? Respir Med 2014; 108: 647-653.

36 Stuart BD, Lee JS, Kozlitina J, et al. Effect of telomere length on survival in patients with idiopathic pulmonary fibrosis: an observational cohort study with independent validation. Lancet Respir Med 2014; 2: 557-565.

37 Blodgett J, Theou O, Kirkland S, et al. Frailty in NHANES: comparing the frailty index and phenotype. Arch Gerontol Geriatr 2015; 60: 464-470.

38 Mitnitski AB, Graham JE, Mogilner AJ, et al. Frailty, fitness and late-life mortality in relation to chronological and biological age. BMC Geriatr 2002; 2: 1. 